\title{
Capacitive pressure sensors based on MEMS, operating in harsh environments
}

\begin{abstract}
Poly-crystalline silicon carbide (polysic) Micro-electromechanical systems (MEMS) capacitive pressure sensors operating at harsh environments (e.g. high temperature) are proposed because of $\mathrm{SiC}$ owing excellent electrical stability, mechanical robustness, and chemical inertness properties. The principle of this paper is, design, simulation. The application of $\mathrm{SiC}$ pressure sensors are in a harsh environments such as automotive industries, aerospace, oil/logging equipments, nuclear station, power station. The sensor demonstrated a high temperature sensing capability up to $400 \mathrm{degC}$, the device achieves a linear characteristic response and consists of a circular clamped-edges poly-sic diaphragm suspended over sealed cavity on a silicon carbide substrate. The sensor is operating in touch mode capacitive pressure sensor, The advantages of a touch mode are the robust structure that make the sensor to withstand harsh environment, near linear output, and large over-range protection, operating in wide range of pressure, higher sensitivity than the near linear operation in normal mode, so in this case some of stray capacitance effects can be neglected.
\end{abstract}

Keyword: MEMS; Touch mode capacitive pressure sensor; High-temperature; Polycrystalline silicon carbide; PSG; Harsh environment 